\title{
BMJ Open Association between early working life patterns, in publicly and privately owned companies, and the course of future sickness absence due to mental disorders: a cohort study in Catalonia (Spain)
}

Amaya Ayala-Garcia (D) ,1,2,3 Laura Serra, ${ }^{1,2,3,4}$ Monica Ubalde-Lopez ${ }^{2,5,6}$

To cite: Ayala-Garcia A, Serra L, Ubalde-Lopez M. Association between early working life patterns, in publicly and privately owned companies, and the course of future sickness absence due to mental disorders: a cohort study in Catalonia (Spain). BMJ Open 2021;11:e040480. doi:10.1136/ bmjopen-2020-040480

- Prepublication history and supplemental material for this paper is available online. To view these files, please visit the journal online (http://dx.doi org/10.1136/bmjopen-2020040480).

Received 17 May 2020 Revised 16 December 2020 Accepted 07 January 2021
D) Check for updates

(c) Author(s) (or their employer(s)) 2021. Re-use permitted under CC BY-NC. No commercial re-use. See rights and permissions. Published by BMJ.

For numbered affiliations see end of article.

\section{Correspondence to} Dr Amaya Ayala-Garcia; amaya.ayala01@estudiant. upf.edu

\section{ABSTRACT}

Objectives To assess the relationship between early working life patterns, at privately and publicly held companies, and the course of sickness absence (SA) due to mental disorders.

Methods Cohort study of workers aged 18-28 years, affiliated with the Spanish social security system, living in Catalonia, who had at least one episode of SA due to mental disorders between 2012 and 2014. Individual prior working life trajectories were reconstructed through sequence analysis. Optimal matching analysis was performed to identify early working life patterns by clustering similar individual trajectories. SA trajectories were identified using latent class growth modelling analysis. Finally, the relationship between early working life patterns and subsequent SA trajectories was assessed via multinomial logistic regression models.

Results Among both men and women, four labour market participation (LMP) patterns were identified: stable permanent employment (reference group), increasing permanent employment, fluctuating employment and delayed employment. Among women, an increasing permanent employment pattern in early working life was related to a decrease of accumulated SA days over time (adjusted OR (aOR) 2.08; 95\% Cl 1.18 to 3.66). In men, we observed a trend towards a middle stable accumulation of $\mathrm{SA}$ days in those with fluctuating employment (aOR $1.25,95 \% \mathrm{Cl} 0.57$ to 2.74 ) or delayed employment (aOR 1.79; $95 \% \mathrm{Cl} 0.59$ to 5.41 ). In both men and women, an early working life in big companies was related to a more favourable SA trajectory.

Conclusions Early LMP patterns characterised by an increasing stability—decreased number of transitions between temporary contracts and lack of social security coverage towards permanent contracts-were related to a better future SA course due to mental diagnosis.

\section{INTRODUCTION}

According to Eurostat, since the 1990s, Spain has been among the European countries with the lowest employment rates $(<70 \%)$. The
Strengths and limitations of this study

- This register-based study was performed using a large administrative database, guaranteeing that the sample represents the Spanish workforce, linked with sickness absence registers, allowing the selection of diagnosis subgroups with a considerable sample size over a 10-year time period.

It is a novel approach to assess how future health may be affected by prior working life transitions, considering employment status, type of contract, unemployment and periods of lack of social security coverage.

- This longitudinal study takes a life-course approach to examining precarious working life, and its effects on future sickness absence due to mental disease.

- A potential classification bias may occur since individuals who shifted among categories of explanatory and adjustment variables over the follow-up period were assigned to the category in which they spent the most time during the follow-up.

- We lacked information regarding prior health status, which could potentially influence future sickness absence and/or eligibility for more precarious employment.

gap between the European and the Spanish average employment rates is largest within the young working population (20\% vs $34 \%$, respectively). Moreover, in 2017, Spain had the highest proportion of persons working as temporary contract employees, and one of the highest rates of precarious employment (having a work contract of only $\leq 3$ months) compared with the European average. ${ }^{1}$

Spain has historically had a core of publicly held companies that are characterised as providing permanent and secure job contracts, workers' protection and trade union membership agreements. ${ }^{2}$ However, 
the phenomena of globalisation, continuous incorporation of women into the labour market, technological developments, organisational requirements, outsourcing and population ageing have contributed to a process of deregulation towards a more flexible labour market and employment relations. Labour market fluctuations have led to alterations of practices inherent to privately held companies, such as revising staffing, contracting and expanding on production expenses. Following the economic crisis in 2008, the recovery of employment rates has been largely based on temporary and partial employments, together with nonstandard forms of employment (ie, informal employment without social security coverage or any type of contract). ${ }^{3}$ In 2009, the rate of temporary contracts in private companies was exceeded by that in public companies ( $25.5 \%$ vs $26.5 \%$ ), and both were equal in $2014(24 \%) .{ }^{2}{ }^{4-6}$ Therefore, the young working force has largely delayed the onset of their working life, mainly entering employment with temporary contracts and low salaries. $^{7}$

Sickness absence (SA) is defined as an absence from work due to a medically certified health-related problem. It is a global measure of health, and can be understood as a tool of social protection in that economic (ie, SA benefits) and medical support are provided from the National Healthcare System during the episode. ${ }^{8}$ In industrialised countries, SA has a huge impact on the healthcare system, with both social and economic expenditures, ${ }^{9-11}$ accounting for $€ 6.6$ billion in Spain in 2018. ${ }^{12}$ The most frequent diagnoses behind SA are musculoskeletal disorders and mental disorders. ${ }^{13}$

Several studies have reported that unemployment, temporary employment and job insecurity are related to mental disorders, stress and poor self-rated health. ${ }^{14}{ }^{15} \mathrm{In}$ 2017, a scoping study highlighted one mechanism that drives the effects of precarious employment and unemployment on mental disorders among young people-namely, the life-course perspective, suggesting a cumulative nature of the exposure. ${ }^{16}$ It has been projected that the frequency of mental disorders will increase, particularly affecting young adults. ${ }^{17}$ The body of evidence regarding SA determinants among workers with mental disorders has been mostly focused on Northern European countries, with fewer studies performed in the Southern European working population. ${ }^{18-20}$ The socioeconomic and cultural contexts differ between these regions, as does the security in the labour market, with the Northern European countries characterised by a flexicurity that ensures reemployment after unemployment based on training, mediation, and reintegration programmes, in addition to generous income replacement. ${ }^{21}$

Health outcomes and work participation clearly differ between men and women and among different age groups ${ }^{22}$ highlighting the need for separate investigations of predictors of SA course. Regarding differences in work participation, precariousness is believed to be a gendered phenomenon due to processes of family formation, gender segregation and wage discrimination, which contribute to an already unstable labour market context. ${ }^{23}$ Existing literature has focused on whether public companies attract potentially absent workers, due to worse health status and greater self-interest, compared with the presumed more restrictive SA practices in the private sector. ${ }^{24-26}$ A study in Finland found that the risk for more adverse SA trajectories did not differ between public and private employees. ${ }^{5}$ However, the relationship between the potential effects of previous working life on SA trajectories in the private and public sectors has generally been poorly studied.

The literature shows a diverse amalgam of approaches to the relationship between health and employment in terms of the prevention of SA due to mental diagnosis. Past research has mainly focused on working conditions, psychosocial factors, lifestyle factors, specific worker groups or employment status and contracts. ${ }^{18} 2728$ Recent research has applied the life-course perspective to labour-market trajectories to assess how working life affects health outcomes, such as SA. Longitudinal analysis provides a more holistic perspective of work participation due to the consideration of transitions, order and duration of employment statuses. ${ }^{149}$

In the present study, we aimed to explore from a lifecourse perspective the relationship between labour market participation (LMP) trajectories at the start of working life, at public and private companies, and the course of future SA due to mental disorders.

\section{METHODS}

We performed a register-based cohort study among 1379 young workers (aged 18-28 years in 2002) who were included in the Spanish WORKss cohort. ${ }^{30}$ All included persons were affiliated with Spanish social security, living in Catalonia and had at least one episode of SA due to a mental diagnosis between 2012 and 2014. The individuals included in the WORKss cohort are part of the Continuous Working Life Sample (CWLS). The age range of 18-28 years was selected in accordance with the objective of the study, which was to evaluate the young population at the start of their working life.

The CWLS was started in 2004, and includes an annual cross-sectional representative sample of $4 \%$ of the population affiliated with Spanish social security. Data available from the CWLS allows reconstruction of working life based on information related to occupational level, employment status/conditions (ie, employment, unemployment, type of contract, salary and working time), social benefits (ie, unemployment, permanent disability and retirement), other work-related variables (ie, company ownership and size) and date of death. Additionally, SA registries were obtained from the Catalan Institute for Medical and Health Evaluations (ICAM by its acronym in Spanish). The provided data included information related to the diagnosis causing an SA episode (coded according to 10th edition of the International 
Classification of Diseases (ICD-10)), as well as the SA starting and ending date. ${ }^{31}$

The acquired registry data were used to reconstruct early working life trajectories from 2002 to 2011, based on transitions between four work-related states: permanent, temporary contract, unemployment and without social security coverage. We also recorded the following secondary explicative variables: early working life trajectory, company ownership (private or public), and company size (small/medium: $\leq 100$ employees; or big: $>100$ employees). Individuals were assigned a company ownership and size based on the category in which they spent most of their early working life.

For the SA trajectory analysis, we measured SA as the number of days accumulated per year due to any medically certified mental disorder between 2012 and 2014. All mental and behavioural disorders (ICD-10, chapter V, F00-F99) were included.

Employment conditions during the SA follow-up period were evaluated as potential confounders. The recorded conditions included occupational category (non-manual skilled, non-manual non-skilled, manual skilled or manual non-skilled), working time (full, part time, short part time or marginal part time), type of contract (permanent or temporary) and annual income (low, medium or high). Workers who changed across categories over time were assigned the category in which they spent most of the follow-up period.

\section{Patient and public involvement statement}

This study was designed and conducted based on secondary administrative records, retrieved from the Spanish social security system and the ICAM. Patients were not involved in any stage of the study. Confidentiality was maintained in both databases. The authors received data that were already anonymised, as specified in a record linkage agreement between Spanish social security, the ICAM and the Centre for Research in Occupational Health-Pompeu Fabra University.

\section{Statistical analysis}

Based on transitions among the above-described four working life states, sequence analysis was performed to reconstruct individual working life. Next, optimal matching analysis was applied, and we identified groups of workers who shared similar working life trajectories, that is, LMP patterns. ${ }^{32}$ Average silhouette width was used to select the optimal number of clusters.

SA trajectories were identified using the statistical technique of latent class growth analysis. In this methodology, an indicator of interest is considered across time points, allowing the identification of homogeneous subgroups that share similar characteristics. Trajectories can be estimated, assuming they follow either a linear quadratic function or any other expression, including more complex elements. Notably, it is recommended to apply the criterion of parsimony, and to use the simplest expression to obtain similar results. ${ }^{33}$ The optimal number of trajectories was assessed considering the lower Bayesian information criterion and the Lo-Mendell-Rubin adjusted and bootstrap likelihood ratio tests. In cases where the compared fit indexes had similar values, the one with the highest entropy was preferentially chosen. ${ }^{34}$ The size of each class was dependent on the sample size and how meaningful a small group was for the study aim.

Finally, to study the association between LMP patterns and SA trajectories, we applied a multinomial logistic regression. All analyses were stratified by sex. R Statistical Software was used for sequence analysis and optimal matching, Stata v.13 software for multinomial regression models and MPlus software for latent class analysis.

\section{RESULTS \\ LMP patterns}

Among both men and women, four LMP patterns were identified: stable permanent employment $(45.6 \%$ and $43.6 \%$ of workers, respectively), increasing permanent employment $(23.7 \%, 20.6 \%)$, fluctuating employment $(18.9 \%, 24.2 \%)$ and delayed employment $(11.8 \%, 11.6 \%)$ (table 1, figure 1). In both sexes, the delayed employment pattern included the youngest workers (mean age of 21.9 years for women and 22.1 for men), while the stable permanent employment pattern included the oldest workers (mean age of 24 years for women and 23.8 for men). The stable permanent employment and increasing permanent employment patterns included the highest proportion of workers with high and medium incomes, especially among men.

In both sexes, the stable permanent employment pattern comprised a steady proportion of about $90 \%$ of workers with permanent contracts (figure 1). Among women, this pattern included the lowest proportions of workers employed at publicly owned companies (7.2\%), part-time jobs (10\%) and manual non-skilled occupations $(5.5 \%)$ compared with the other patterns.

Half of the individuals showing the increasing permanent employment pattern were workers on temporary contracts who slowly switched to permanent contracts over time, reaching up to $90 \%$ by the end of follow-up (figure 1). Compared with other patterns, this pattern included higher proportions of women $(38.5 \%)$ and men $(30.1 \%)$ employed by large companies, and of women in part-time jobs (18.3\%). The increasing permanent employment pattern included less skilled occupations among men (20.4\%) compared with women (6.3\%).

Within the delayed employment pattern, we identified a group of young workers who gradually entered into the labour market with permanent (up to $40 \%$ of individuals) and temporary contracts (20\%) (figure 1). Women in this pattern had the greatest proportions of marginal parttime jobs (2.9\%) and manual non-skilled jobs (15.4\%), and men in this pattern had the highest proportion of part-time jobs (8.6\%); however, these patterns were based on very small numbers of individuals. 


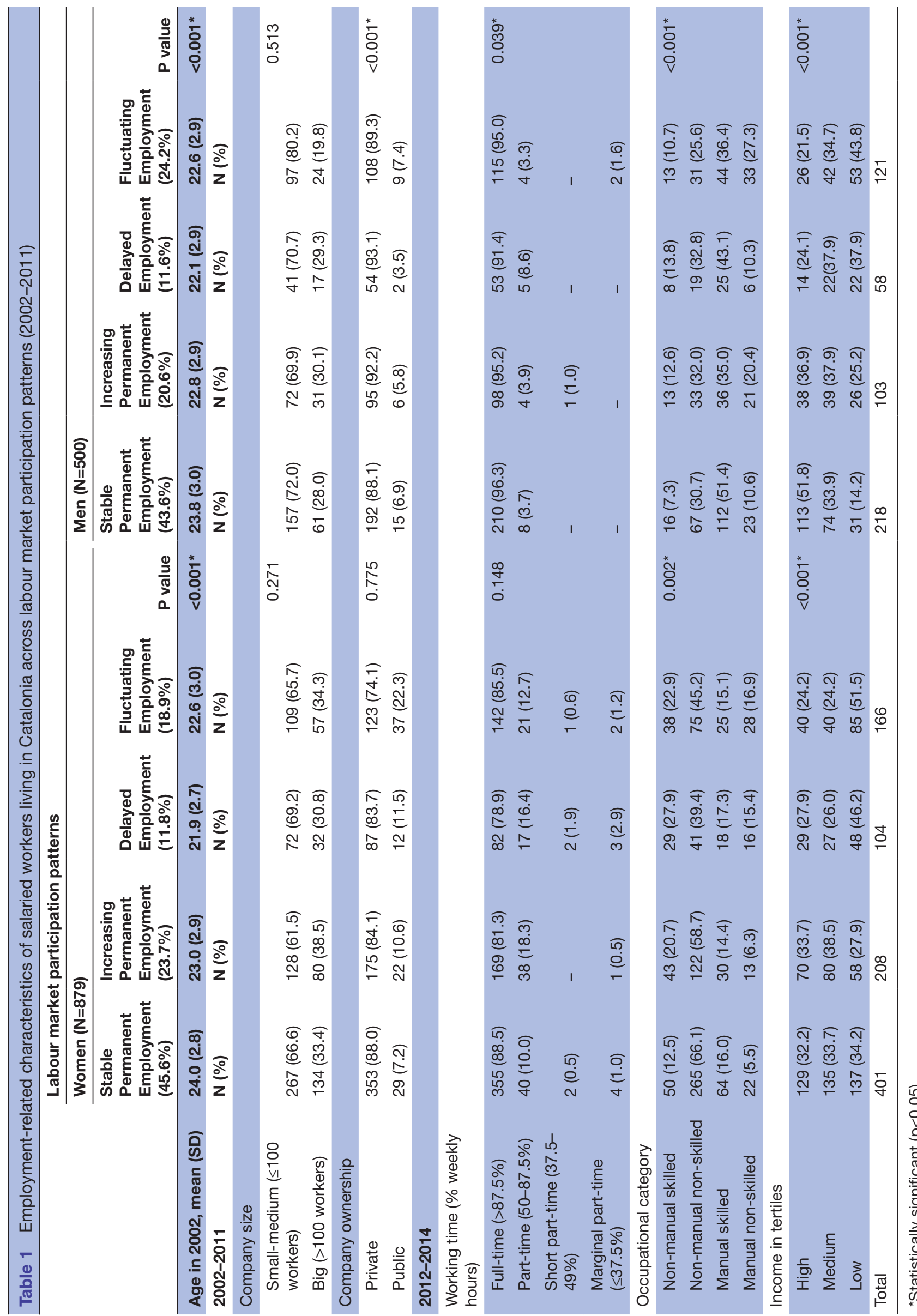


Women

$(n=879)$
Men

$(n=500)$
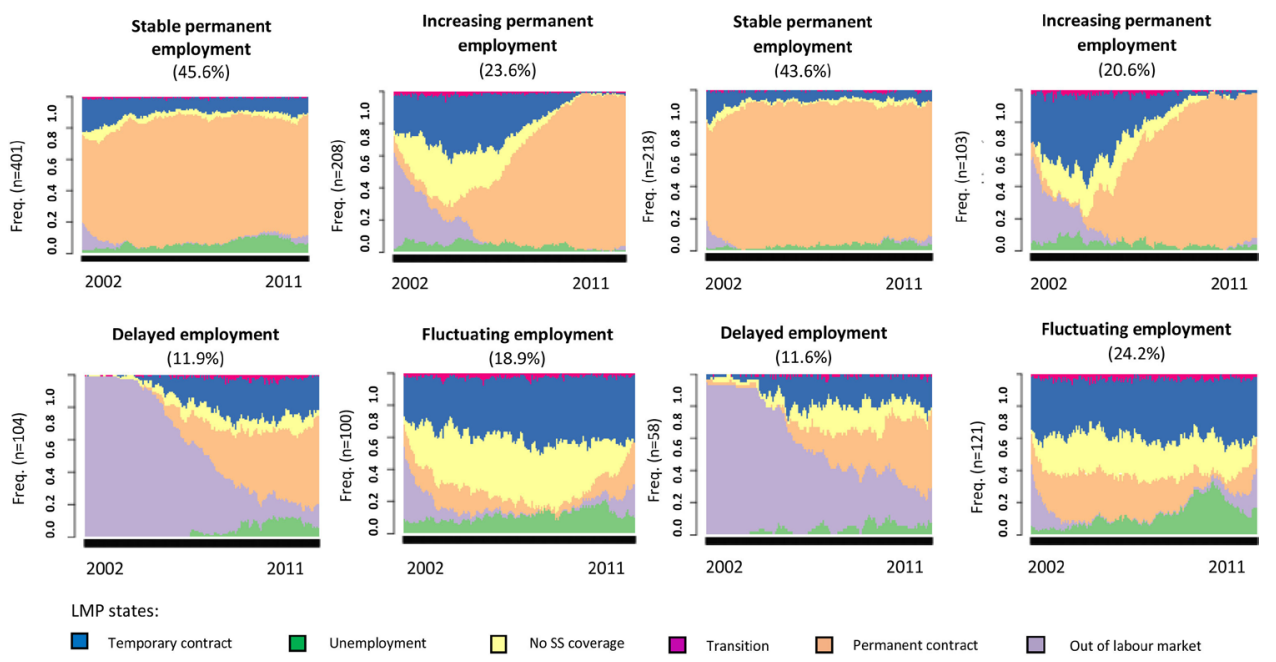

Figure 1 Early labour market participation (LMP) patterns in salaried workers living in Catalonia (2002-2011).

Finally, the fluctuating employment pattern presented an unsteady working life, switching from a high proportion of temporary contracts $(40 \%)$ to unemployment $(15 \%)$ and periods without social security coverage $(40 \%)$ (figure 1). Among both women and men, this pattern included the highest proportions of young workers employed by publicly owned companies $(22.3 \%$ and $7.4 \%$, respectively), in manual non-skilled occupations $(16.9 \%$ and $27.3 \%)$, and with the lowest salary levels (51.5\% and $43.8 \%)$.

\section{SA trajectories}

Men and women exhibited different SA trajectories due to mental disorders. Among women, we identified four trajectories of accumulated days of SA per year: low stable (40.6\% of workers), low decreasing (27.5\%), decreasing $(14.7 \%)$ and increasing (17.2\%) (online supplemental figure 1). The low stable SA trajectory was characterised by the lowest accumulation of days during the follow-up period (median duration: 6-15 days), but the highest proportion of episodes $(38.2 \%)$. The decreasing trajectory showed the lowest number of episodes $(16.4 \%)$ and represented the smallest proportion of workers (14.7\%), but accumulated a high number of days annually (median duration: 38.5-97 days). In contrast, the increasing trajectory stood out as including a large proportion of women with temporary contracts $(25.8 \%)$, who generated $28.1 \%$ of episodes (table 2).

Among men, three SA trajectories were identified: low stable $(55.2 \%$ workers), middle stable $(31 \%)$ and high stable (13.8\%) (online supplemental figure 2). Compared with the other two SA trajectories, the low stable trajectory included a larger proportion of workers with high income levels $(40.9 \%)$, the lowest proportion of manual non-skilled occupations $(14.5 \%)$ and a lower proportion of temporary contracts $(14.5 \%)$. This trajectory had the lowest annual accumulation of days on SA (median duration: 6-11 days), but the highest proportion of episodes $(52.1 \%)$. In contrast, the high stable trajectory included $13.8 \%$ of workers and represented the highest proportion of low-income levels (44.9\%). The high stable trajectory showed the lowest number of episodes $(13.9 \%)$ but the highest accumulated days (median duration: 123.5-194 days) (table 3).

\section{Association between early LMP patterns and SA trajectories}

Each person in the sample suffered from a mental disease at some point, and thus the probabilities must be interpreted accordingly. Increasing or decreasing risk was determined based on a more favourable, though never a desirable, SA trajectory. Multinomial logistic regression analysis showed that compared with women with a stable permanent LMP pattern, women with an increasing permanent employment LMP pattern were more likely to exhibit a decreased risk of accumulating days on a future SA due to mental disorders (adjusted OR, aOR 2.08; 95\% CI 1.18 to 3.66) (table 4). Among women, being predominantly employed by a big company, compared with a small-medium company, was associated with an increased probability of developing a future low stable SA trajectory rather than a low decreasing trajectory (aOR $0.64 ; 95 \%$ CI 0.45 to 0.93 ) or a decreasing trajectory (aOR $0.46 ; 95 \%$ CI 0.28 to 0.74 ). Additionally, among women, an early working life that was mainly developed within publicly owned companies, compared with privately owned companies, was related to a future low decreasing SA trajectory (aOR 1.81; 95\% CI 1.01 to 3.25). Finally, analysis of the predominant type of contract during the SA follow-up period revealed that women who mainly held temporary jobs, compared with those predominantly in permanent jobs, exhibited a higher probability of increasing accumulated days on SA (aOR 1.98; 95\% CI 1.10 to 3.55 ) (table 4 ). 


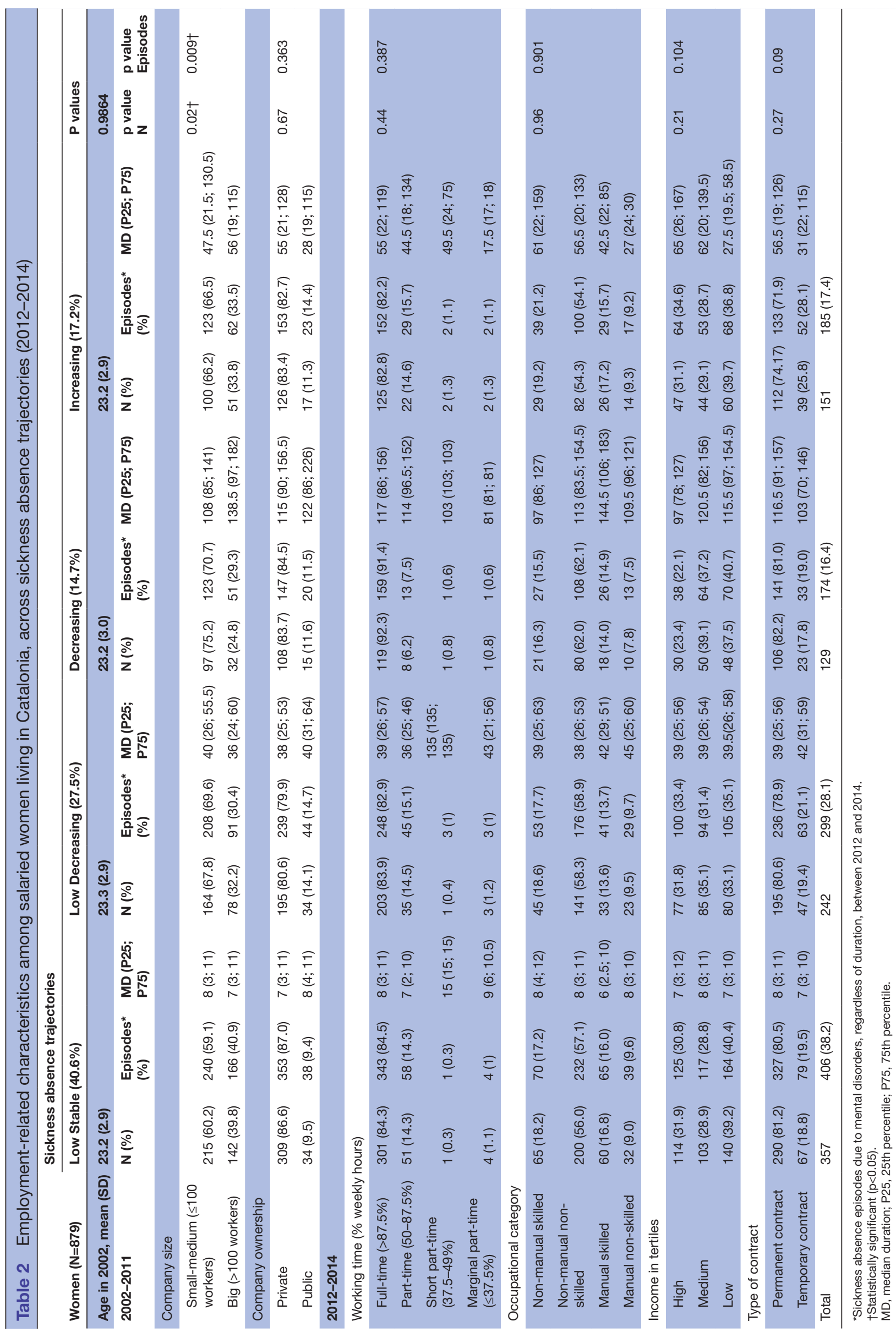




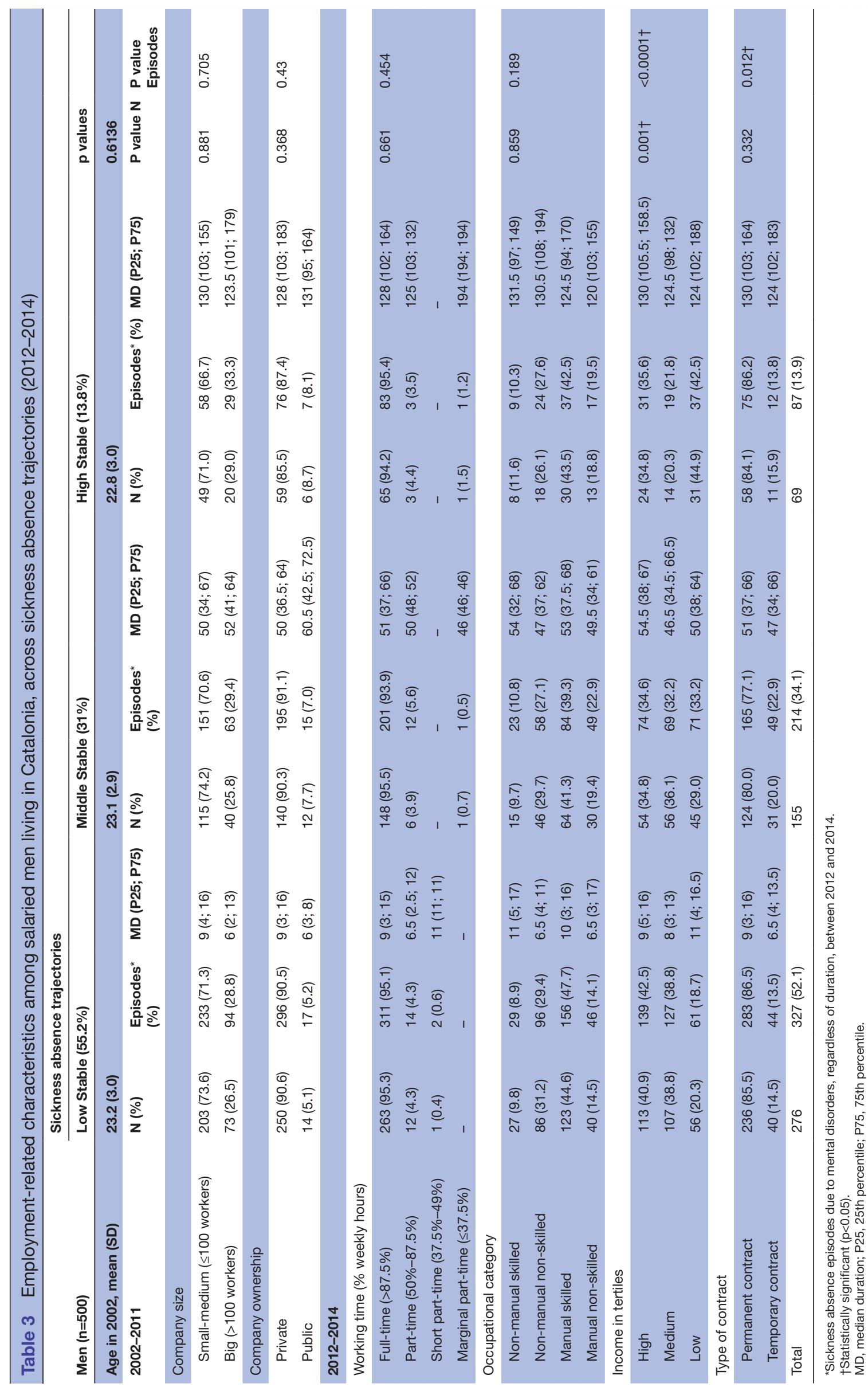




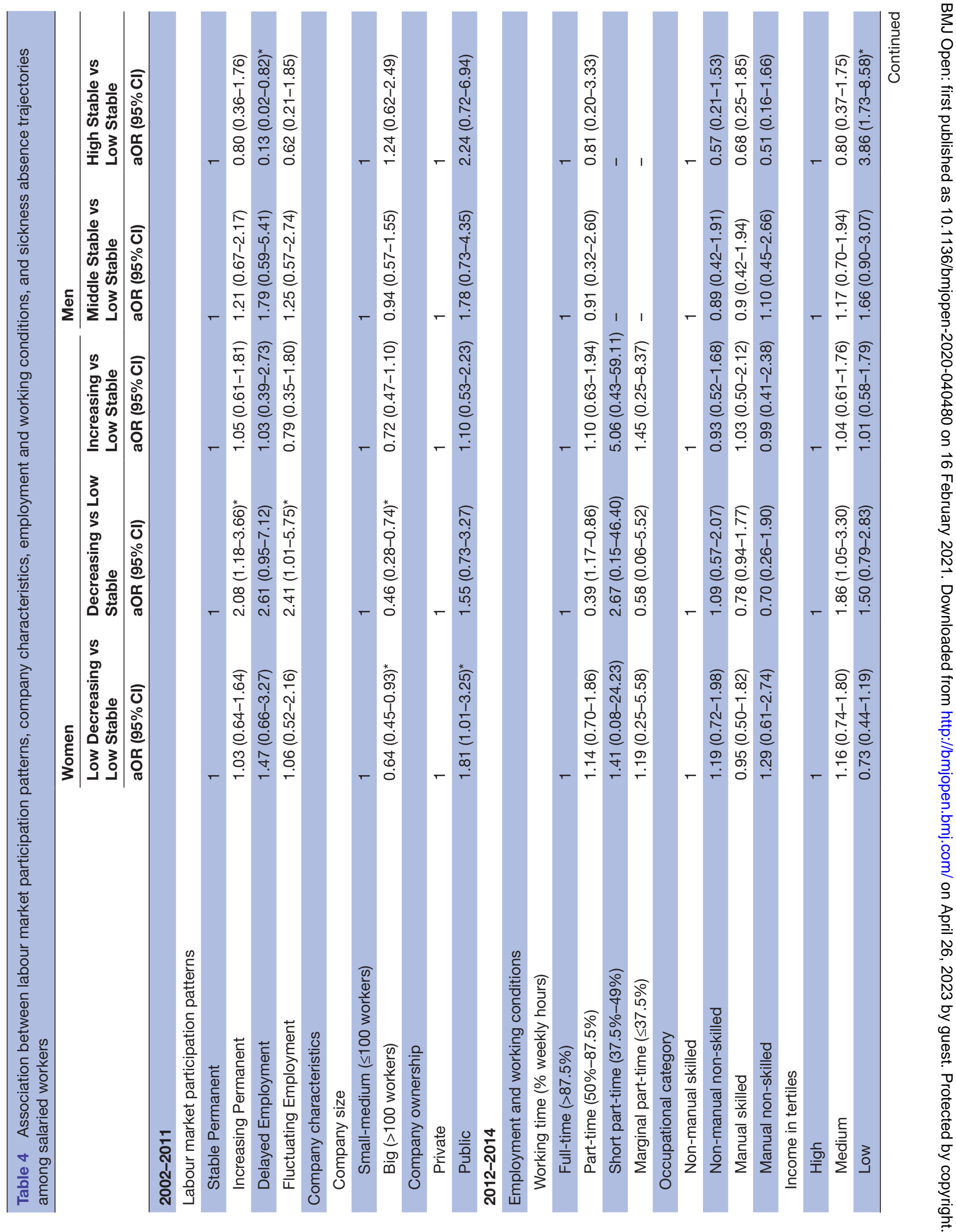




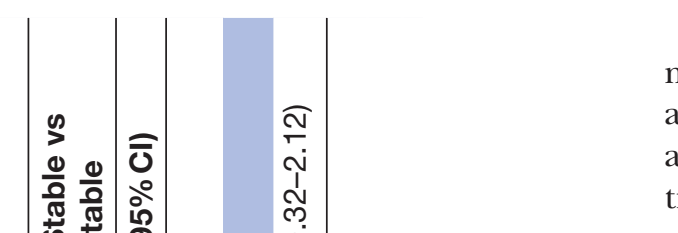

Among men, we found that showing a delayed employment LMP, compared with a stable employment LMP, was associated with a higher probability of having a low stable accumulation of SA days rather than a high stable SA trajectory (aOR 0.13 ; $95 \%$ CI 0.02 to 0.84 ). In men, low salary levels were strongly related to a higher probability of a future high stable SA trajectory (aOR 3.86; 95\% CI 1.73 to 8.58 ), and showed a trend of association with a middle stable SA trajectory although it was not statistically significant (aOR $1.66 ; 95 \%$ CI 0.90 to 3.07 ).

\section{DISCUSSION}

In this study, we assessed the role of early LMP patterns in the later course of SA due to mental diagnosis within a sample of salaried workers in Spain. Women whose early working life followed an increasing permanent employment pattern were more likely to exhibit a decrease of accumulated SA days over time. Among men, an early increasing permanent, fluctuating employment or delayed employment pattern showed a trend of association with a future middle stable accumulation of SA days rather than a low stable pattern. Women whose early working life was mainly developed in big companies were more likely to exhibit a more favourable course of future SA. In contrast, women who were more often employed at public companies showed an increased risk of an adverse course of mental health-related absence.

The identified patterns of LMP were in line with what would be expected for a working population at the beginning of their working life. Young adults tend to enter the labour market lacking both work experience and appropriate working opportunities, ${ }^{16}$ and their entrance might be delayed due to the extended training periods that correspond with younger ages. Notably, we observed a fluctuating pattern, indicating that young adults experienced constant transitions-switching between temporary contracts, unemployment and periods without social security coverage. Stable and increasing permanent employment patterns were associated with the oldest workers in our cohort, who exhibited a high proportion of permanent contracts.

Our present analysis of LMP patterns revealed that part-time jobs were more prevalent among women compared with men (online supplemental table 1). This higher prevalence of part-time work among women has long been characteristic in older adults. ${ }^{35}$ Moreover, higher proportions of part-time employment were found in workers who had a delayed entrance to employment or who steadily got permanent employment, such that the extension of part-time work to the younger population could result from the combination of studying and working. However, in Spain, over half of part-time jobs are reported to be involuntary. ${ }^{3}$ Notably, in the previous literature, the voluntariness of part-time work and social class are reported to be determinants of the potential harmful effect on mental health, ${ }^{35}{ }^{36}$ related to the implied reduction of income. 
We hypothesised that more transitions during early working life would be associated with higher risk of an adverse course of SA due to mental disorders. The patterns that most depicted a precarious early working life were fluctuating employment and delayed employment, and thus, we expected that persons with these patterns would more commonly show a worse course of future SA for mental disorders. Our results revealed differences between men and women, although these findings should be cautiously interpreted since some working life patterns were exhibited by a small number of workers, potentially leading to non-significant associations. Men with fluctuating or delayed early employment patterns tended to show a middle stable rather than a low stable future accumulation of SA days, whereas women exhibited a tendency towards decreasing days on SA. Nevertheless, among men, a delayed entry to the labour market showed a later protective effect against a course of accumulating a high number of SA days. This finding could potentially be related to avoidance of the risk of a precarious labour market through family support, as reported in the literature, which enables the young population to delay entry into the labour market, attain a higher education level, and potentially access more favourable employment. ${ }^{37}$ However, this hypothesis is not confirmed in women. Hence, there may also be a gender-based explanation related to access to better contracts. Notably, our results are inconclusive in terms of significance, and thus, the observed associations should be interpreted as trends.

In the current literature, there is a lack of prior evidence regarding how transitions during early working life affect the course of future mental health. Some studies claim that being employed with a temporary contract is more often a choice based on previous mental health status, while others demonstrate a relationship between employment-unemployment transitions and poor mental health. ${ }^{36} 38{ }^{39}$ Moreover, return-to-work expectations are reportedly lower among workers with mental conditions compared with those with physical limitations due to the symptoms of mental health conditions, such as hopelessness, low self-efficacy and insecurity, ${ }^{40}$ or due to stigmatisation or lack of support at work. ${ }^{18}$ These studies have mostly use self-reported health measures and a cross-sectional approach to assess the effects of poor mental health on future employment. Additionally, a few longitudinal studies have assessed the effect of ageing, family trajectories, or occupational category on work attachment. ${ }^{41} \mathrm{~A}$ life-course perspective has been applied to examine how mental health trajectories in young adults might impact work, but such studies have measured work as a single event, assessing the effect of pre-existing mental disorders or considering the longitudinal approach for return to work. ${ }^{42}{ }^{43}$ The potential effects of transitions within the labour market on future SA remain unexplored.

Individuals whose working life mainly involved employment at publicly owned companies were more likely to later accumulate a higher number of SA days. This association could be related to the fact that workers at publicly owned companies feel more confident about using such benefits, more supported when asking for SA or less pressured to prematurely return to work. Some studies claim that such an association could be driven by the selection of low-motivation workers or people with health-related problems or other limitations into this sector, due to the belief that these working conditions are more suitable for them. ${ }^{24-26}$

The SA trajectories with accumulation of fewer SA days were more likely to include workers from big companies than from small-to-medium companies. This could suggest that big companies may be a more facilitating and less stigmatising setting when claiming an SA. Moreover, in smaller companies, employees tend to have specific roles that cannot be easily replaced due to reliance and shortage of resources to replace staff, and this setting may foster a stronger sense of compromise. ${ }^{44}$

It is important to note that our study coincided with the economic crisis, which led to structural changes in the labour market. This context could have favoured future presentism. Additionally, there could be a selection bias in terms of healthy workers, further aggravated by the economic recession. ${ }^{45}$ In this setting, the fear of not being renewed or not finding a new job in the labour patterns showing the greatest transitions could be related to a later high accumulation of SA days.

The main limitation of the study was the assignment of individuals to the explanatory variables and to the categories of the adjustment variables. Many individuals shifted among categories over the follow-up period, and in these cases, we assigned them to the category in which they spent most of their time during the follow-up. This could have led to a misclassification bias that might underestimate the accumulation of SA days in other categories. Furthermore, we could not account for the effects of prior health status on the course of SA as this information was not available. This may have led to overestimating results, since people with worse prior mental health could be more prone to have developed worse LMP trajectories. It is also important to note that our results do not represent the whole public sector since, in Spain, civil servants with permanent contracts follow different healthcare schemes. Likewise, the methodology applied to the LMP patterns and SA trajectories involved group-based analyses that classified individuals according to similar behaviours. Thus, some of the resulting groups had a very small number of observations, and these results should be interpreted with caution. However, some authors argue that a minimum of $5 \%$ should be enough to consider a pattern, and our results are above these recommendations. ${ }^{46}$

Our study also had several strengths. It was conducted using a large administrative database that guaranteed representativeness of the sample, allowing us to select diagnosis subgroups with a considerable sample size, and with a lengthy time window of 10 years. Moreover, it was a novel approach to determine employment status based on type of contract (temporary and permanent), and periods of unemployment and lack of social security 
coverage, and to assess the transitions between these states. The diagnoses causing SA episodes were medically certified by primary doctors rather than self-reported, enhancing the validity of our results. Finally, the changes in the public sector cannot be overlooked, and our study provides insight into the differences from the private sector.

\section{CONCLUSION}

Overall, our present results provide insights regarding how labour market transitions-characterised by employment flexibility and high unemployment rates-have impacted the course of future mental health among the youngest working population.

Our analyses revealed that early working lives characterised by transitions between types of contracts, and periods of unemployment and lack of social security coverage, were apparently related to a worse mental health course. Future public health policies should consider how a precarious labour market can shape the course of mental health in young working populations, with the aim of addressing and preventing SA in the long term.

\author{
Author affiliations \\ ${ }^{1}$ Center for Research in Occupational Health (CiSAL), Universitat Pompeu Fabra \\ (UPF), Barcelona, Spain \\ ${ }^{2}$ CIBER of Epidemiology and Public Health (CIBERESP), Madrid, Spain \\ ${ }^{3}$ IMIM - Parc Salut Mar, Barcelona, Spain \\ ${ }^{4}$ Research Group on Statistics, Econometrics and Health (GRECS), University of \\ Girona, Girona, Spain \\ ${ }^{5}$ Barcelona Institute for Global Health (ISGlobal), Barcelona, Spain \\ ${ }^{6}$ Universitat Pompeu Fabra (UPF), Barcelona, Spain
}

Acknowledgements This work wouldn't have been possible without the Spanish National Social Security Institute and the Catalonian Institute for Medical Evaluations.

Contributors All listed authors fulfil authorship criteria. MUL and LS participated in the study conception and design. LS and AA-G performed the data management and analysis. AA-G, MUL and LS interpreted the data. AA-G drafted the first version of the manuscript with close help from MUL. MUL and LS made subsequent revisions to the manuscript, and all of the authors revised the final version, agreed with the text and findings, and approved this final version. The corresponding author certifies that all listed authors meet authorship criteria. AA-G is the guarantor.

Funding The study was financed by the State Plan for Investigation, Development, and Innovation, 2013-2016, by the Health Institute Carlos III-FIS_FEDER Subdirection General of Evaluation and Promotion of Investigation (Grants FIS $\mathrm{P} I 17 / 00220)$.

Competing interests None declared.

Patient consent for publication Not required.

Ethics approval This study was performed in accordance with the standards of Good Clinical Practice and the principles of the Declaration of Helsinki. The study protocol guaranteed the fulfilment of Regulation (EU) 2016/679 of the European Parliament and the Council of 27 April 2016 on the protection of natural persons regarding the processing of personal data and the free movement of such data. It also fulfilled the Spanish Organic Law 3/2018 of 5 December on the Protection of Personal Data and the Guarantee of Digital Rights. This study was approved by the Parc de Salut Mar Ethics Committee in Barcelona (Research Protocol no 2019/8506/I). The research team committed itself to the strict use of data for the present study. Additionally, a linkage protocol agreement between the Centre for Research in Occupational Health at the Pompeu Fabra University, the National Social Security Institute, and the Catalonian Institute for Medical Evaluations guaranteed the maintenance of confidentiality in providing the identified datasets to the authors.

Provenance and peer review Not commissioned; externally peer reviewed.

Data availability statement Data may be obtained from a third party and are not publicly available. Data are not available in a repository. Administrative data comes from an annual sample of Spanish Social Security affiliates that are part of the Continuous Working Life Sample (CWLS), provided by the General Directorate of Social Security (DGOSS by its acronym in Spanish) and linked records of sickness absence episodes certified in Catalonia provided by Catalan Institute for Medical and Health Evaluations (ICAM by its acronym in Catalan). The records linkage has been possible thanks to an agreement signed between DGOSS, ICAM and CiSALUPF, maintaining data confidentiality.

Supplemental material This content has been supplied by the author(s). It has not been vetted by BMJ Publishing Group Limited (BMJ) and may not have been peer-reviewed. Any opinions or recommendations discussed are solely those of the author(s) and are not endorsed by BMJ. BMJ disclaims all liability and responsibility arising from any reliance placed on the content. Where the content includes any translated material, BMJ does not warrant the accuracy and reliability of the translations (including but not limited to local regulations, clinical guidelines, terminology, drug names and drug dosages), and is not responsible for any error and/or omissions arising from translation and adaptation or otherwise.

Open access This is an open access article distributed in accordance with the Creative Commons Attribution Non Commercial (CC BY-NC 4.0) license, which permits others to distribute, remix, adapt, build upon this work non-commercially, and license their derivative works on different terms, provided the original work is properly cited, appropriate credit is given, any changes made indicated, and the use is non-commercial. See: http://creativecommons.org/licenses/by-nc/4.0/.

Author note MUL's main affiliation is ISGlobal.

ORCID iD

Amaya Ayala-Garcia http://orcid.org/0000-0002-0415-8177

\section{REFERENCES}

1 Eurostat. Temporary and part-time jobs on the rise - Product Eurostat [online]. Products Eurostat News, 2018. Available: https:// ec.europa.eu/eurostat/web/products-eurostat-news/-/WDN20180813-1 [Accessed 22 Nov 2018].

2 Pitxer Campos J V, Sánchez Velasco A, Lorente Campos Adoración Guamán Hernández R. La dinámica de la temporalidad en el mercado laboral español en las dos últimas crisis: cambios normativos, modelo productivo y prácticas empresariales [online]. Valladolid, 2014. Available: http://webs.ucm.es/info/ec/jec14/ comunica/A_EL/A_EL_2.pdf [Accessed 22 Oct 2018].

3 OIT. Non-standard employment around the world: Understanding challenges, shaping prospects [online], 2016. Available: http://www. ilo.org/wcmsp5/groups/public/-dgreports/-dcomm/-publ/documents/ publication/wcms_534326.pdf

4 Fernández Gutiérrez M. Empleo público Y temporalidad: ¿es El sector público parte del problema? Temas Laborales, Cons Andaluz Relac Laborales [Internet] 2009;102:51-87 http://www.academia.edu/ 5505972/Empleo_público_y_temporalidad._Es_el_sector_público_ parte_del_problema

5 Virtanen P, Siukola A, Lipiäinen L, et al. Trajectory analyses of sickness absence among industrial and municipal employees. Occup Med 2017;67:109-13.

6 Instituto Nacional de Estadística. National Statistics Institute [online]. Available: http://www.ine.es/dynt3/inebase/index.htm?padre=982\& capsel=985 [Accessed 30 Apr 2019].

7 Dirección de Estadística y Análisis Sociolaboral. Informe Jóvenes y Mercado de Trabajo [online], 2018. Available: http://www.empleo. gob.es/es/sec trabajo/analisis-mercado- [Accessed 22 Oct 2018].

8 Delclós J, García S, López JC. Duración de la incapacidad temporal POR contingencia común POR grupos diagnósticos duration of non work-related sickness absence by clinical diagnosis. Arch Prev Riesgos Labor 2010;13:180-7.

9 OCDE. Factsheet on promoting mental health [online], 2018. Available: http://www.oecd.org/health/health-systems/OECDFactsheet-Mental-Health-Health-at-a-Glance-Europe-2018.pdf [Accessed 22 Apr 2019].

10 Gabbay M, Taylor L, Sheppard L, et al. NICE guidance on long-term sickness and incapacity. Br J Gen Pract 2011;61:e118-24.

11 WHO. European Health Information Gateway. Absenteeism from work due to illness, days per employee per year - European Health 
Information Gateway [online], 2018. Available: https://gateway.euro. who.int/en/indicators/hfa_411-2700-absenteeism-from-work-due-toillness-days-per-employee-per-year/ [Accessed 22 Apr 2019].

12 Ministerio de Trabajo MYSS. Sistema de la seguridad social. Resumen de Ejecución del Presupuesto, 2018.

13 Finnes A, Enebrink P, Ghaderi A, et al. Psychological treatments for return to work in individuals on sickness absence due to common mental disorders or musculoskeletal disorders: a systematic review and meta-analysis of randomized-controlled trials. Int Arch Occup Environ Health 2019;92:273-93.

14 Lallukka T, Kronholm E, Pekkala J, et al. Work participation trajectories among 1,098,748 Finns: reasons for premature labour market exit and the incidence of sickness absence due to mental disorders and musculoskeletal diseases. BMC Public Health 2019:19:1418.

15 Canivet C, Bodin T, Emmelin M, et al. Precarious employment is a risk factor for poor mental health in young individuals in Sweden: a cohort study with multiple follow-ups. BMC Public Health 2016;16:687.

16 Vancea M, Utzet M. How unemployment and precarious employment affect the health of young people: a scoping study on social determinants. Scand J Public Health 2017;45:73-84.

17 Patel V, Flisher AJ, Hetrick S, et al. Mental health of young people: a global public-health challenge. The Lancet 2007;369:1302-13.

18 de Vries H, Fishta A, Weikert B, et al. Determinants of sickness absence and return to work among employees with common mental disorders: a scoping review. J Occup Rehabil 2018;28:393-417.

19 Norder G, Roelen CAM, Bültmann U, et al. Shift work and mental health sickness absence: a 10-year observational cohort study among male production workers. Scand $J$ Work Environ Health 2015:41:413-6.

20 Norder G, Roelen CAM, van der Klink JJL, et al. External validation and update of a prediction rule for the duration of sickness absence due to common mental disorders. J Occup Rehabil 2017;27:202-9.

21 Wilthagen T, Tros F. The concept of 'flexicurity': a new approach to regulating employment and labour markets. Transfer 2004;10:166-86.

22 Kerkhofs M, Lindeboom M. Age related health dynamics and changes in labour market status. Health Econ 1996;1997:407-23.

23 Stuth S, Jahn K. Young, successful, precarious? precariousness at the entry stage of employment careers in Germany. J Youth Stud 2020;23:702-25.

24 Mastekaasa A. Absenteeism in the public and the private sector: does the public sector attract high absence employees. J Public Adm Res Theory. 2019:1-17.

25 Böhm T, Riedel N. On selection into public civil service. CESifo Economic Studies 2013;59:34-71.

26 Dur R, Zoutenbier R. Intrinsic motivations of public sector employees: evidence for Germany. Ger Econ Rev 2015;16:343-66.

27 Virtanen M, Ervasti J, Head J, et al. Lifestyle factors and risk of sickness absence from work: a multicohort study. Lancet Public Health 2018;3:e545-54.

28 Amick BC, McLeod CB, Bültmann U. Labor markets and health: an integrated life course perspective. Scand J Work Environ Health 2016;42:346-53.

29 Ervasti J, Kausto J, Koskinen A, et al. Labor market participation before and after long-term part-time sickness absence in Finland: a population-based cohort study. J Occup Environ Med 2020;62:E142-8.
30 Gómez MAL, Durán X, Zaballa E. Cohort profile: the Spanish working life social security (WORKss) cohort study. BMJ Open 2016;6.

31 Durán A. La Muestra Continua de Vidas Laborales de la Seguridad Social. Rev del Minist Trab y Asuntos Soc [online], 2007. Available: http://www.mitramiss.gob.es/es/publica/pub_electronicas/ destacadas/revista/numeros/ExtraSS07/Est09.pdf [Accessed 24 Oct 2018].

32 Abbott A, Tsay A. Sequence analysis and optimal matching methods in sociology: review and prospect. Sociological Methods and Research 2000;29:3-33.

33 Skrondal A, Sophia R-H. Generalized latent variable modeling statistical analysis of shapes clinical trials in oncology second edition design and analysis of quality of life studies in clinical trials dynamical search introduction to measurement error and Bayesian adjustments St. New York, 2004.

34 van de Schoot R, Sijbrandij M, Winter SD, et al. The GRoLTSChecklist: guidelines for reporting on latent trajectory studies. Struct Equat Model : Multidiscipl J 2017;24:451-67.

35 Barnay T. Health, work and working conditions: a review of the European economic literature. Eur J Health Econ 2016;17:693-709.

36 Waenerlund A-K, Virtanen P, Hammarström A. Is temporary employment related to health status? analysis of the Northern Swedish cohort. Scand J Public Health 2011;39:533-9.

37 Serracant P. The impact of the economic crisis on youth trajectories. Young 2015;23:39-58.

38 Dawson C, Veliziotis M, Pacheco G, et al. Is temporary employment a cause or consequence of poor mental health? a panel data analysis. Soc Sci Med 2015;134:50-8.

39 Virtanen M, Kivimäki M, Joensuu M, et al. Temporary employment and health: a review. Int J Epidemiol 2005;34:610-22.

40 Pedersen $\mathrm{P}$, Lund T, Lindholdt L, et al. Labour market trajectories following sickness absence due to self-reported all cause morbidity-a longitudinal study. BMC Public Health 2016;16:1-10.

41 Lu W, Benson R, Glaser K, et al. Relationship between employment histories and frailty trajectories in later life: evidence from the English longitudinal study of ageing. $J$ Epidemiol Community Health 2017;71:439-45.

42 Norder G, van der Ben CA, Roelen CAM, et al. Beyond return to work from sickness absence due to mental disorders: 5-year longitudinal study of employment status among production workers. Eur J Public Health 2017;27:79-83.

43 Arends I, Almansa J, Stansfeld SA, et al. One-year trajectories of mental health and work outcomes post return to work in patients with common mental disorders. J Affect Disord 2019;257:263-70.

44 Alba Ramirez A. La incapacidad temporal para el trabajo: análisis económico de su incidencia y su duración [online], 2009. Available: http://www.seg-social.es/wps/wcm/connect/wss/f4e8cb57-4ffO4c03-abbf-3003665f18a3/F9_07.pdf?MOD=AJPERES\&CVID= [Accessed 5 May 2019].

45 Leinonen T, Viikari-Juntura E, Husgafvel-Pursiainen K, et al. Cause-Specific sickness absence trends by occupational class and industrial sector in the context of recent labour market changes: a Finnish panel data study. BMJ Open 2018;8:e019822-11.

46 Farrants K, Friberg E, Sjölund S, et al. Trajectories of future sickness absence and disability pension days among individuals with a new sickness absence spell due to osteoarthritis diagnosis $\geq 21$ days: a prospective cohort study with 13-month follow-up. BMJ Open 2019;9:e030054-11. 